\title{
Mechanisms Leading to an Acute Rise in Intraocular Pressure in Retinal Vein Occlusion
}

\author{
ALEC M ANSONS, LOUIS CLEARKIN, IAIN BRYCE \\ Liverpool
}

\begin{abstract}
Summary
Forty patients with retinal vein occlusion were studied to investigate possible mechanisms leading to an acute rise in intraocular pressure. A rise in intraocular pressure on recumbency was found in $64 \%$ of eyes with a central retinal vein occlusion and $65 \%$ of eyes with a branch retinal vein occlusion. This finding correlated with glucose tolerance test score, and may be the result of abnormal glucose tolerance in these patients.

Following provocative testing using pilocarpine and phenylephrine, no eye showed irido-corneal contact or angle closure, however over $50 \%$ of eyes release pigment into the anterior chamber. Fifty-two per cent of central retinal vein occlusion and $71 \%$ of branch retinal vein occlusion eyes that released pigment showed a rise in intraocular pressure.
\end{abstract}

The pathogenesis of retinal vein occlusion is multifactorial, ${ }^{1}$ clinical and experimental evidence has stressed the role of reduced retinal perfusion. ${ }^{1-3}$ Patients who have suffered a retinal vein occlusion have been shown to have reduced retinal artery pressure on ophthalmodynometry, ${ }^{3}$ and in experimental animals the ischaemic type of retinal vein occlusion could only be produced if the retinal artery was occluded. ${ }^{4}$ Retinal artery perfusion pressure may be reduced by either a fall in systemic blood pressure or a rise in intraocular pressure (IOP). This prompted us to look at three possible mechanisms in which acute elevations in IOP may occur in patients with retinal vein occlusion. We have assessed the posturally induced change in IOP, angle closure 'risk' with pilocarpine and phenylephrine ( $\mathrm{P} \& \mathrm{P})$ provocative testing, and also pigment release into the anterior chamber with subsequent rise in IOP following provocative testing.

\section{Materials}

Patients who had suffered a retinal vein occlusion and were attending the out-patients clinics of St Paul's Eye Hospital Liverpool during the months of January to May 1984 were recruited to the study. These patients were required to attend on several occasions to have the tests performed. Any patient who was unable to fulfil this requirement was not included. Patients who were taking drugs known to affect IOP were excluded from the study. None of the patients entering the study were known diabetics. All patients gave informed consent, and ethical committee approval had been obtained.

An age and sex matched group of subjects for the standing and lying IOP part of the study were recruited as controls.

Patients were divided into three groups:

Group 1. Twelve men and eight women who had suffered a CRVO. Their ages ranged from 41 to 79 years, (mean $=64$ years). The

From: St Paul's Eye Hospital, Old Hall Street, Liverpool L3 9PF.

Correspondence to: Mr A M Ansons, St Paul's Eye Hospital, Old Hall Street, Liverpool L3 9PF. 
time from onset of symptoms to entry into the study ranged from two days to 26 weeks, (mean $=7.5$ weeks). One patient had had his fellow eye enucleated as a child.

Group 2. Ten men and ten women who had suffered a BRVO. Their ages ranged from 37 to 71 years, $($ mean $=61$ years $)$, and the time from onset of symptoms to entry into the study ranged from one week to 52 weeks, (mean $=17.3$ weeks).

Group 3. Ten men and ten women control subjects with no history of eye disease or general medical condition, recruited from relatives of patients with minor complaints attending the eye hospital. Their ages ranged from 32 years to 77 years $($ mean $=62$ years).

\section{Methods}

Each subject underwent the following investigations.

(1) Postural change in IOP was measured.

After instillation of topical anaesthetic and fluorescein dye, the patient was asked to stand upright, breathing normally, and IOP was measured in both eyes using a Perkins hand held applanation tonometer, the mean of three readings was recorded. The subject was then asked to lie on a couch and after two minutes in the supine position the IOP was again measured in both eyes in a similar fashion.

\section{(2) Slit lamp biomicroscopy}

Using a Haag Streit 900 slit lamp, the anterior segment of the eye was examined. Particular attention was paid to the presence of lens capsular pseudoexfoliation, iris transillumination defects, pigmentary changes in the pupil margin or a Kruchenberg spindle. Central anterior chamber depth was then measured using a Goldmann Pachometer.

(3) Pilocarpine and phenylephrine provocative testing

Initial IOP was recorded using a Goldmann applanation tonometer. Following this 2\% pilocarpine and $10 \%$ phenylephrine (P\&P) drops were simultaneously instilled, one drop of each was instilled into each eye every minute for three applications. At 30 minute intervals for the next two hours IOP was measured and the anterior segment examination repeated, and the presence of any pigment in the aqueous recorded. Instillation of $\mathrm{P} \& \mathrm{P}$ was repeated in the fashion described above at the end of the first hour. At the end of the second hour the study was terminated with the instillation of $0.5 \%$ thymoxamine drops.

\section{(4) Glucose tolerance test}

On a separate occasion, following an overnight fast, the patient was asked to drink $50 \mathrm{~g}$ of glucose. Blood was taken for serum glucose estimation before and two hours after ingestion of the glucose.

A two hour blood glucose level of less than $6.1 \mathrm{mmol} / \mathrm{l}$ was taken as normal, 6.1$10 \mathrm{mmol} / \mathrm{l}$ showed impaired glucose tolerance and greater than $10 \mathrm{mmol} / \mathrm{l}$ indicated diabetes.

\section{Results}

\section{Intraocular pressure}

Baseline IOPs recorded in the sitting position prior to P\&P testing showed the IOP of the affected eye to be lower than the fellow eye in $53 \%$ of CRVO patients and $40 \%$ of BRVO patients.

In patients with CRVO the IOP levels in affected eyes were significantly lower than fellow eyes (paired $\mathrm{t}=2.42, \mathrm{p}<0.05$ ), whereas the IOPs did not signficantly differ in patients with BRVO (paired $\mathrm{t}=1.56, \mathrm{p}=0.13$ ). In the control subjects there was no significant inter-eye IOP difference (paired $\mathrm{t}=0.75$, $p=0.46)$. There was a low correlation between inter-eye IOP difference and duration of occlusion for both CRVO patients $(\mathrm{r}=0.44, \mathrm{p}=0.06)$ and BRVO patients $(\mathrm{r}=0.09, \mathrm{p}=0.72)$.

Eight eyes (from 5 patients) in the CRVO group and only one eye in the BRVO group had an IOP of greater than $21 \mathrm{mmHg}$. None of the controls had an IOP greater than $21 \mathrm{mmHg}$.

The change in IOP induced by recumbency was calculated by subtracting the standing IOP from the lying IOP measurement. The results are shown in Figure 1. In the retinal vein occlusion group there was a significantly greater IOP rise induced by recumbency than controls.

In the CRVO group the mean rise in IOP was significantly greater in both affected and fellow eyes compared with controls. The mean rise in affected eyes was $3.4 \pm 2.4$ (SD) $\mathrm{mmHg}$ (unpaired $\mathrm{t}=5.96, \mathrm{p}<0.0001$ ) and in the fellow eyes $3.7 \pm 3$ (SD) $\mathrm{mmHg}$ (unpaired 


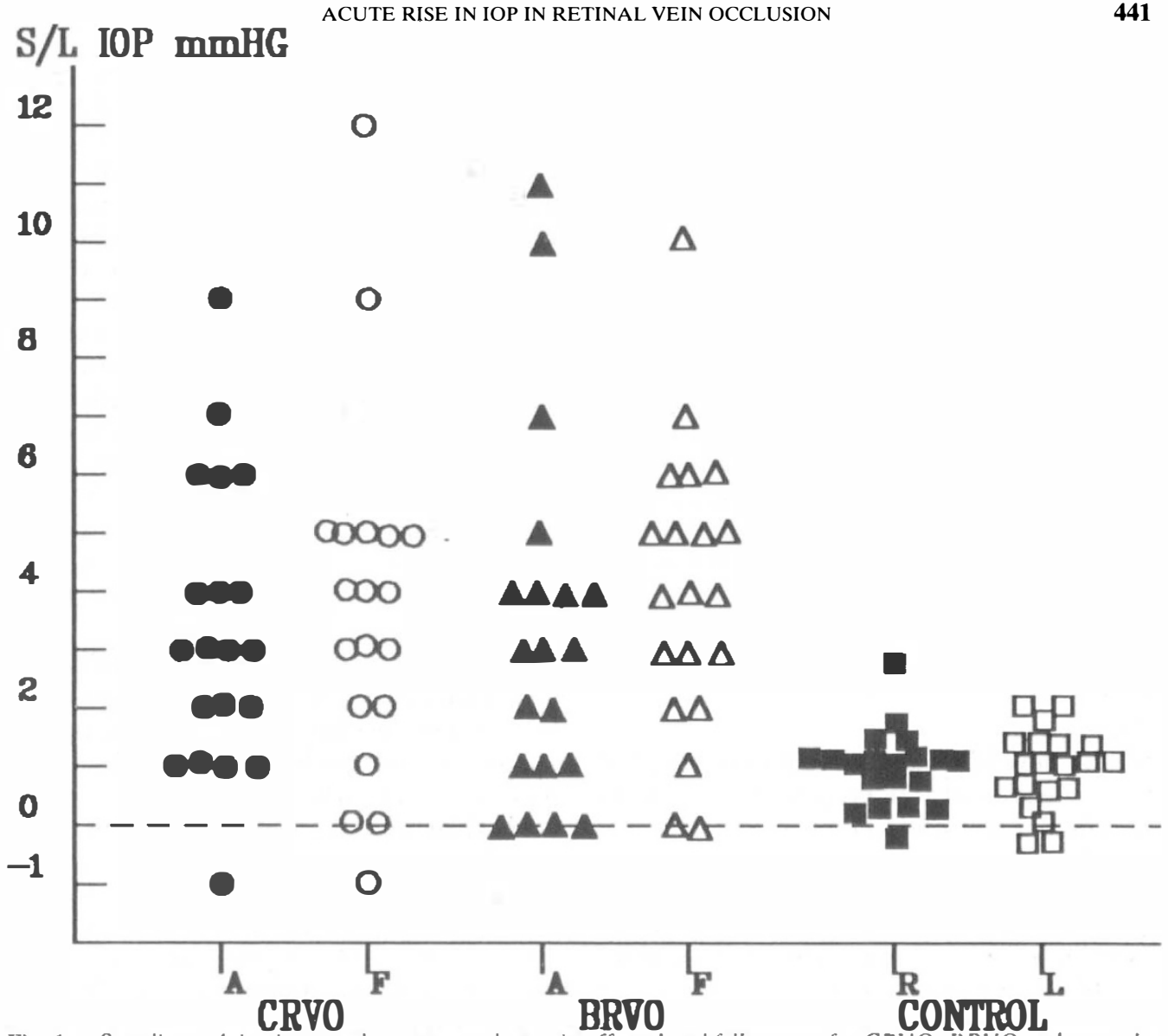

Fig. 1. Standing to lying intraocular pressure change in affected and fellow eyes for CRVO, BRVO and controls. $A=$ affected eye, $F=$ fellow eye, $R=$ right eye, $L=$ left eye, $S / L=$ standing $/$ lying.

$\mathrm{t}=5.64, \mathrm{p}<0.0001)$. In the BRVO the mean rise in IOP was also significantly greater in both affected and fellow eyes compared with controls. The mean rise in affected eyes was $3.3 \pm 3.0$ (SD) $\mathrm{mmHg}$ (unpaired $\mathrm{t}=4.59$, $\mathrm{p}<0.0001)$ and in fellow eyes $4.1 \pm 2.4(\mathrm{SD})$ mmHg (unpaired $\mathrm{t}=7.72, \mathrm{p}<0.0001$ ). There was no significant difference for IOP rise between affected and fellow eyes for either the CRVO or BRVO groups.

There was a low correlation between the postural IOP change in affected and fellow eyes in both CRVO $(\mathrm{r}=0.33, \mathrm{p}=0.17)$ and BRVO $(r=0.39, p=0.09)$ groups. On the other hand, the postural IOP change in right and left control group eyes was closely correlated $(\mathrm{r}=0.63, \mathrm{p}=0.003)$.

Glucose tolerance test

Two out of 20 patients in the CRVO group and one out of 20 patients in the BRVO group were found to be diabetic, and three patients in the CRVO group and four patients in the BRVO group had impaired glucose tolerance.

A correlation of postural IOP change was sought with glucose tolerance, duration of occlusion and age. A high correlation was only found for glucose tolerance test results with both CRVO fellow eyes $(r=0.6$, $\mathrm{p}=0.014)$ and BRVO affected eyes $(r=0.66, p=0.002)$. After pooling the affected eyes and fellow eyes postural IOP change from both vein occlusion groups and correlating these two pooled groups with glucose tolerance test scores a significant correlation for affected eyes $(r=0.387, p=0.014)$ and fellow eyes $(r=0.463, p=0.003)$ was found.

Anterior chamber depth

The anterior chamber depths for CRVO 
patients ranged from $2.1 \mathrm{~mm}$ to $3.2 \mathrm{~mm}$ $($ mean $=2.6 \mathrm{~mm})$ in the affected eye and $2 \mathrm{~mm}$ to $3.4 \mathrm{~mm}$ (mean $=2.6 \mathrm{~mm}$ ) in the fellow eye. The BRVO patients ranged from $1.9 \mathrm{~mm}$ to $3.2 \mathrm{~mm}$ (mean $=2.5 \mathrm{~mm}$ ) in the affected eye and $2 \mathrm{~mm}$ to $3.3 \mathrm{~mm}$ $($ mean $=2.5 \mathrm{~mm})$ in the fellow eye.

\section{Anterior segment examination}

Forty-eight eyes (61\%) (22/39 in the CRVO group and 26/40 in the BRVO group) had anomalies in the pigment layer of the iris. Pigment fall-out in the collarette was the most frequent anomaly noted, a large number had small areas of iris transillumination defects and only one patient (in the CRVO group) had a Kruckenberg spindle. No patient had pseudoexfoliation.

\section{Provocative testing with Pilocarpine and Phenylephrine}

No eye developed a positive provocative test (an evaluation of IOP greater than $8 \mathrm{~mm} \mathrm{Hg}$ or irido-corneal contact).

Following provocative testing $65 \%$ of affected eyes and $63 \%$ of fellow eyes in the CRVO group showed substantial pigment release into the anterior chamber. The BRVO group showed a similar response with $55 \%$ of affected and $60 \%$ of fellow eyes releasing pigment into the anterior chamber. In nearly all cases the eye had previously been noted to have iris pigment anomalies.

The results of provocative testing in relation to pigment release and IOP change are shown in Figure 2. Overall 52\% of CRVO eyes and $71 \%$ of BRVO eyes that released pigment showed a rise in IOP.

The patients in the CRVO group who released pigment showed a significantly greater IOP rise compared with those who did not (unpaired $\mathrm{t}=2.07, \mathrm{p}<0.05$ ). A similar difference was shown for BRVO patients (unpaired $t=2.51, p<0.05$ ). Three out of the 39 patients showed a unilateral release of pigment (excluding the CRVO patient with only one eye).

\section{Discussion}

Vannas in $1960^{5}$ reported five cases of angle closure glaucoma associated with retinal vein occlusion. In three of the cases the retinal vein occlusion was diagnosed at the time of the acute attack. Although provocative testing was performed, it was limited to those patients who gonioscopically did not have narrow angles, or in whom there were no records of previous acute attacks. One finding known to be associated with angle closure glaucoma is a shallow anterior chamber. Our results show that the anterior chamber depths of patients wtih retinal vein occlusion lie within the normal range for age matched controls, ${ }^{6}$ and outside the range of patients with angle closure. The result of provocative testing wtih $P \& P$ was negative in all patients. We feel therefore that there is unlikely to be an association or causal relationship between angle closure (pupil block) and retinal vein occlusion.

Anomalies in the iris pigment epithelium were found in 48/79 retinal vein occlusion eyes $(61 \%)$. The incidence of this in the normal population has not been defined. They may represent areas where pigment has already been shed into the anterior chamber, this speculation is further supported by the finding of pigment release into the anterior chamber on provocative testing with $\mathrm{P} \& \mathrm{P}$ in patients who also showed iris pigment anomalies. Pigment release was found in over $50 \%$ of affected and fellow eyes, the incidence in normal subjects varies between $5 \%$ and $27 \%$. ${ }^{7}$ The mean IOP rise in patients releasing pigment was significantly greater than those who did not release pigment. Pigment as it leaves the anterior chamber may block the trabecular meshwork causing an acute rise in IOP, especially if the facility for outflow is already compromised. ${ }^{8}$ Williams et al. ${ }^{9}$ when testing for a relationship between outflow facility and abnormal postural rise in IOP, found outflow facility to be reduced in some patients with retinal vein occlusion, although they failed to demonstrate any association between the two. Despite this, differences in outflow facility may have been a factor why not all our patients who released pigment showed a rise in IOP, although outflow facility was not measured in our group. The presence or absence of pigment release was bilateral in $36 / 39$ patients (excluding the patient with only one eye). This suggests that the phenomenon of pigment release probably predated the ret- 


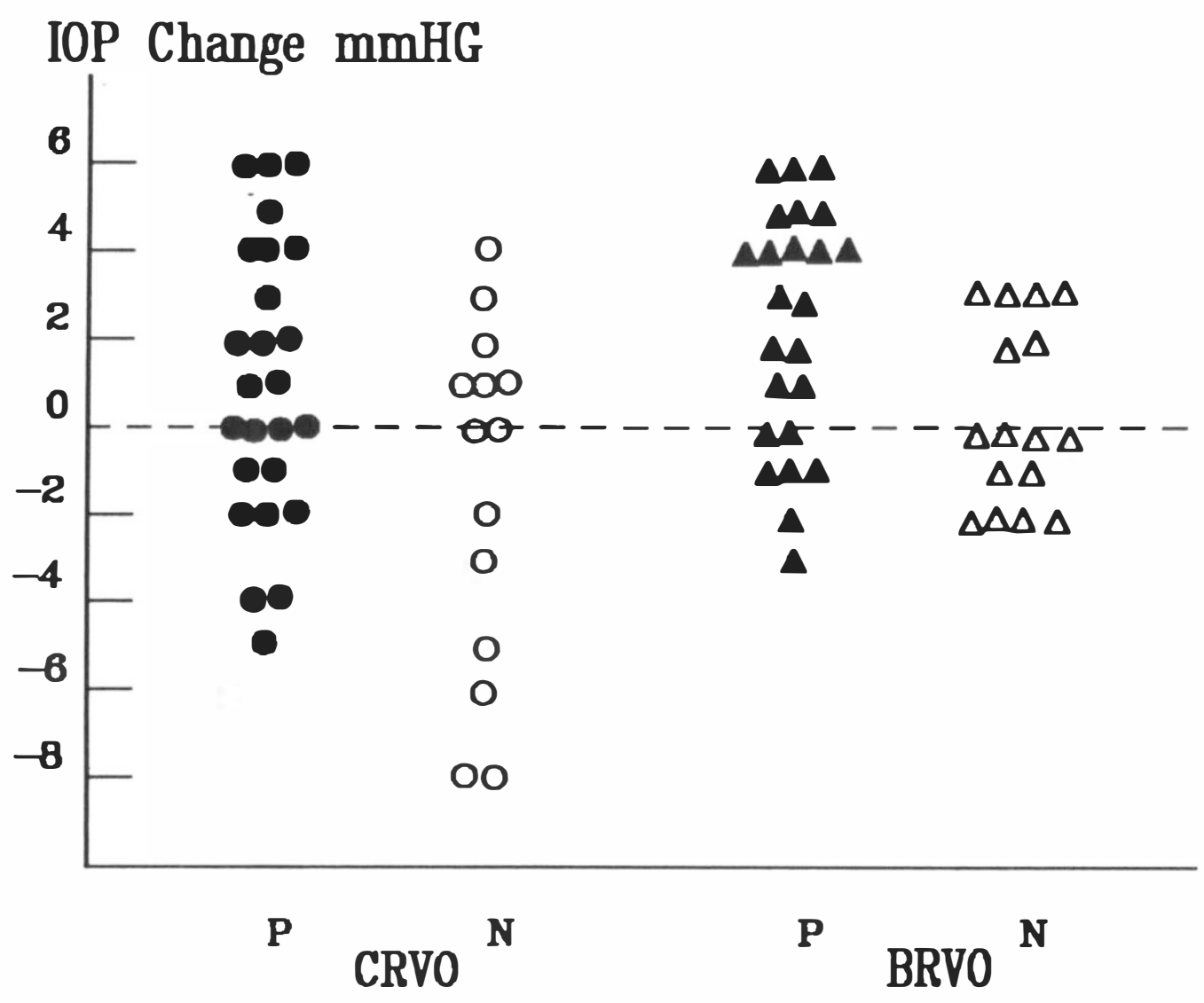

Fig. 2. Intraocular pressure change following provocative testing with $P \& P$ in eyes releasing pigment and eyes not releasing pigment into the anterior chamber. $P=$ pigment release, $N=$ no pigment release.

inal vein occlusion. Cases of spontaneous release of pigment in response to exercise, ${ }^{10}$ dim illumination or emotion, ${ }^{11}$ which when these conditions were duplicated experimentally caused an increase in IOP, are well known. Although provoking the release of pigment is not physiological, it was found to be associated with anomalies in the pigment layer of the iris suggesting these patients had already demonstrated pigment release in the past. Whether the anomalies of the iris pigment epithelium observed in our patients represents the previous loss of pigment in intermittent showers, perhaps associated with a rise in IOP, or a continuous loss of small amounts is not known.

It has been previously reported that in CRVO the IOP in the affected eye may be lower than the fellow eye, the mechanisms proposed for this are speculative ${ }^{12}$ and our results confirm this observation but do not suggest any explanation for this finding.

We confirmed the finding that patients with retinal vein occlusion have a greater elevation in IOP on recumbency than normal subjects. ${ }^{12,14}$ The underlying mechanism is probably one of impaired auto-regulation and it is thought that on recumbency episcleral venous pressure becomes elevated, with consequent elevation in IOP. It is interesting that both eyes of patients with retinal vein occlusion show a rise in IOP on recumbency. This suggests that the abnormal finding probably predated the occlusive rather than was a consequence of it. This breakdown in homeostatic control is further reinforced by the low 
correlation found for IOP change on recumbency between eyes in the retinal vein occlusion patients against the high correlation found in the controls. However, the high correlation between postural IOP change and glucose tolerance test score suggests that this abnormal finding may result from the abnormal glucose tolerance found in this study group.

Our results suggest two possible mechanisms by which IOP may rise in retinal vein occlusion. Firstly on recumbency and secondly from pigment release. The bilaterality of these responses suggests that they probably predate the occlusion, therefore may have been important in setting in motion the train of events leading to the retinal vein occlusion.

We thank Dr. J. Green, Statistics and Computational Maths Department, University of Liverpool for his help with the statistics.

\footnotetext{
References

${ }^{1}$ Hayreh SS: Central retinal vein occlusion. In Mausolf FA ed. The eye in systemic disease. CV Mosby, St Louis, 1980.

${ }^{2}$ Sanders MD and Graham EM: Medical ophthalmology. In Davidson SI ed. Recent Advances in Opthalmology No.6, Churchill Livingstone Edinburgh 1983.

${ }^{3}$ Paton A, Rubenstein K, Smith VH: Arterial insuf-
}

ficiency in retinal venous occlusion. Trans Ophthalmol Soc UK 1964, 66: 559-86.

${ }^{4}$ Hayreh SS, van Heuven AJ, Hayreh MS: Experimental retinal vascular occlusion. 1. Pathogenesis of central retinal vein occlusion. Arch Ophthalmol 1978, 96: 311-23.

${ }^{5}$ Vannas S and Tarkkanen A: Retinal vein occlusion and glaucoma. Tonographic study of the incidence of glaucoma and of its prognostic significance. Br J Opthalmol 1960, 44: 583-9.

${ }^{6}$ Fontana ST and Brubaker RF: Volume and depth of the anterior chamber in the normal aging human eye. Arch Ophthalmol 1980, 98: 1803-8.

${ }^{7}$ Aggarwal JL and Beveridge B: Liberation of the iris pigment in the anterior chamber. Br J Opthalmol 1971, 55: 544-9.

${ }^{8}$ Mapstone R: Pigment release. $\mathrm{Br} \mathrm{J}$ Ophthalmol 1981, 65: 258-63.

${ }^{9}$ Williams BI and Peart WS: Retinal vein obstruction and intraocualr pressure: abnormal postural response independent of facility of outflow. $\mathrm{Br} \mathrm{J}$ Opthamol 1979, 63: 805-7.

${ }^{10}$ Schenker HI, Luntz MH, Kelsb, et al.: Exercise induced increase of intraocular pressure in the pigmentary dispersion syndrome. Am J Ophthalmol 1980, 89: 598-600.

${ }^{11}$ Epstein DL, Boger WP, Grant WM: Phenylephrine provocative testing in the pigmentary dispersion syndrome. Am J Ophthalmol 1978, 85: 43-50.

${ }^{12}$ Hayreh SS, March W, Phelps CD: Ocular hypotony after retinal vascular occlusion. Tr Ophthalmol Soc UK 1977, 97: 757-67.

${ }^{13}$ Anomalous postural response of intraocular pressure in vascular disease (Editorial). Lancet 1981, i: 314 .

${ }^{14}$ Williams BI and Peart WS: Effect of posture on the intraocular pressure of patients with retinal vein occlusion. Br J Ophthalmol 1978, 62: 688-93. 